\title{
A case of preshaped Y-stent technique application to a distal left main bifurcation lesion
}

\author{
Takayuki Onishi ${ }^{1}$ and Tetsuya Tobaru ${ }^{1}$ \\ ${ }^{1}$ Kawasaki Saiwai Hospital
}

October 30, 2020

\begin{abstract}
We performed coronary intervention for a distal left main bifurcation lesion using a preshaped Y-stent technique. The technique, which we herein propose for the first time, comprises two parts: ex vivo stent preparation and subsequent modified Y-stenting using the prepared stents.
\end{abstract}

\section{A case of preshaped Y-stent technique application to a distal left main bifurcation lesion}

Takayuki Onishi, MD, and Tetsuya Tobaru, MD, PhD

Department of Cardiology, Kawasaki Heart Center, Kawasaki Saiwai Hospital

Correspondence Takayuki Onishi, Department of Cardiology, Kawasaki Heart Center, Kawasaki Saiwai Hospital, 31-27 Omiyacho, Saiwai Ward, Kawasaki, Kanagawa 212-0014, Japan, Email: takayuki.onishi@yahoo.com

Preshaped Y-stent for a bifurcation lesion

Word count: 2566

Keywords

Percutaneous coronary intervention, coronary artery disease, bifurcation stenting, Y-stent technique

Key Clinical Message

The preshaped Y-stent technique which comprises ex vivo stent preparation and a subsequent modified Y-stent technique using the prepared stents decreases the complexity of the conventional Y-stent technique.

\section{Abstract}

We performed coronary intervention for a distal left main bifurcation lesion using a preshaped Y-stent technique. The technique, which we herein propose for the first time, comprises two parts: ex vivo stent preparation and subsequent modified Y-stenting using the prepared stents. We dilated the proximal part of the side branch stent, passed a stylet from the most distal dilated strut to the proximal stent ostium, delivered the main branch stent on the stylet from the proximal stent ostium of the dilated side branch stent through the strut at the stylet exit and remounted the dilated portion of the side branch stent onto the stent balloon. Then, we delivered the prepared Y-stent system to the distal left main bifurcation lesion and deployed the side branch stent and the main branch stent consecutively, which was followed by proximal optimization therapy, rewiring of the side branch stent, and the final kissing balloon technique, all leading to an acceptable angiographic result. The preshaped Y-stent technique omits several steps of the conventional Y-stent procedure, reducing procedural complexity and decreasing the incidence of complications. The 
proposed stent preparation can possibly contribute to the development of a novel interventional device for the treatment of bifurcation lesions.

\section{Introduction}

The elective two-stent technique is an indispensable strategy for a very complex bifurcation lesion whose side branch (SB) vessel is too significant to be abandoned, is vulnerable to occlusion by main vessel (MV) stenting due to a heavy plaque burden at the SB ostium and is highly inaccessible due to its complex anatomy. As an upfront two-stent technique, the latest European guideline recommends reverse provisional stenting, Tstenting, culotte and double kissing crush, stating that the choice of strategy depends on the anatomy and size of the vessel, a need for stenting the SB first and operator proficiency(1). Of these, the culotte stenting or Y-stent technique has been a widespread strategy to treat true bifurcation lesions. The conventional Y-stent technique, however, requires complex procedures, including repeated passages of guidewires and a balloon catheter via a stent strut as well as multiple proximal optimization techniques (POT), which could increase the fluoroscopic time, radiation dose and even the complication rate.

We propose a modified version of culotte stenting, namely, the preshaped Y-stent technique, to lessen the complexity of the procedure. The preshaped $Y$-stent technique comprises two parts: ex vivo stent preparation and a subsequent modified Y-stent technique using the prepared stents. We herein demonstrate a case of a left main bifurcation lesion effectively treated using the preshaped Y-stent technique.

\section{Case Series}

An 84-year-old woman with a history of abdominal stent graft implantation underwent percutaneous coronary intervention for a left main distal bifurcation lesion. Due to the shallow angulation of the distal bifurcation in addition to severe stenosis at both the ostia of the left anterior descending artery (LAD) and left circumflex artery (LCX), we planned an elective two-stent strategy using a modified culotte technique. We performed the intervention via the right femoral artery using an 8Fr SPB3.5 guiding catheter (Hyperion, Asahi Intecc, Aichi, Japan). After passing a guidewire (Hi-Torque Balance Middleweight Universal, Abbott Vascular, CA, USA) into the LAD followed by a guidewire (Sion, Asahi Intecc, Aichi, Japan) crossing into the LCX, an intravascular ultrasound (IVUS) (Altaview, Terumo, Tokyo, Japan) revealed a distal reference luminal diameter of $4.0 \mathrm{~mm}$ for the LCX lesion and one measuring $3.5 \mathrm{~mm}$ for the LAD. Therefore, we decided to deploy a 3.5-20 mm Synergy stent (Boston Scientific, MA, USA) for LAD and a 4.0-20 mm Synergy stent (Boston Scientific, MA, USA) for LCX. In this bifurcation lesion in which the diameter of the LCX was larger than that of the LAD, we chose the LCX as the main branch and the LAD as the SB. Before mounting the stents onto the guidewire, we shaped the stents into Y-like figures (Figure 1, Videos 1 and 2). First, we pulled a plastic tube cover a distance of $5 \mathrm{~mm}$ over the stent for the LAD, which we estimated was equal to the length of the left main part of the Y-stent (Supplementary Figure 1). Next, we gradually inflated the stent system with the plastic tube firmly held by hand to prevent movement of the tube on the stent (Supplementary Figure 2). Six-atmosphere inflation was enough to dilate the uncovered stent struts outside the plastic tube (Supplementary Figure 3). Then, we passed a stylet from the most distal stent strut (Supplementary Figure 3, arrow) through the proximal stent ostium (Supplementary Figure 3, arrowhead). Fourth, the stent for the LCX was delivered on the stylet from the ostium of the dilated LAD stent (Supplementary Figure 4 and Supplementary Figure 5, arrowhead) through the strut at the stylet exit (Supplementary Figure 5, arrow). Finally, we gently remounted the dilated portion of the LAD stent on the stent balloon (Supplementary Figure 6). At the completion of the ex vivo Y-stent-reshaping preparation, we put the stent system on both the LAD and the LCX guidewires simultaneously, slowly delivering the stent system to the distal left main bifurcation lesion (Figure 2, Video 3). We first placed the LAD stent (Figure 3) and then deployed the LCX stent (Figure 4), smoothly completing the formation of the Y-like configuration (Video 4). Next, we conducted proximal optimization treatment at the left main trunk using a 5.0 - $10 \mathrm{~mm}$ balloon (Sapphire NC, OrbusNeich, Hong Kong, China) (Figure 5). Then, we passed a guidewire through a stent strut of the dilated LCX stent at the ostium of the LAD, which was then dilated with a 2.0-15 mm balloon (Sapphire II, OrbusNeich, Hong Kong, China) followed by a 4.5-13 mm balloon (Raiden, Kaneka, Tokyo, Japan). After stenting the proximal LAD lesion using a 2.75-20 mm Synergy stent (Boston 
Scientific, MA, USA), we finally performed kissing balloon inflation using two 3.5-15 mm balloons (Sapphire II, OrbusNeich, Hong Kong, China) (Figure 6). We finished the procedure after obtaining an acceptable final angiography (Figure 7) and confirmed the well-apposed stent struts with IVUS at the LAD and LCX ostia and left main trunk (Supplementary Figure 7, 8 and 9, respectively).

\section{Discussion}

We demonstrated a case of a left main distal bifurcation lesion successfully treated with a preshaped Y-stent technique, that is, ex vivo preparation of a two-stent system into a Y-like configuration. To the best of our knowledge, this is the first report showing the feasibility of the preshaped Y-stent strategy in the human coronary artery.

The major advantage of the preshaped Y-stent technique is to omit several steps of the conventional Y-stent procedure. It enables us to eliminate the time and labor associated with the initial steps of the conventional Y-stent procedure, guidewire recrossing through the strut of the first stent, strut dilation with a balloon and second stent system delivery through the dilated strut. The advantage not only reduces radiation and contrast media but also lessens the procedural complexity, possibly leading to a decreased risk of complications.

A bifurcation lesion comprising ostial stenosis of the significant SB with shallow angulation is the lesion where the preshaped Y-stent technique is preferably applied because the SB ostium is definitely dilated with the stent struts. As shown in the present case, some distal left main diseases and proximal LAD diagonal bifurcation lesions are possibly appropriate for the strategy.

The provisional SB stenting strategy, that is, performance of MV stenting first, followed by POT, and only finalized with SB stenting if needed, is currently considered the standard approach for the treatment of the vast majority of bifurcation lesions(1). Previous randomized comparison studies and systematic reviews and meta-analyses have consistently demonstrated favorable outcomes of the one-stent strategy compared with the two-stent approach in terms of efficacy and safety(2-4). Therefore, we should deliberate the application of the Y-stent strategy for bifurcation lesions by judging the significance of the SB vessels, the probability of SB compromise based on the location and amount of plaque, and SB accessibility and angulation.

A possible disadvantage of the preshaped Y-stent technique is that the new carina created by this stenting technique may contrast with the true carina, making it difficult to cross a guidewire or a balloon catheter through the newly created ostial strut and increasing the amount of metal at the newly created bifurcation. To overcome these possible issues, we need to position the preshaped bifurcation as close to the original carina as possible before applying the technique. Furthermore, we must review the stenting results using intravascular imaging, especially with optical coherence tomography, which allows us to assess the structural relationship of the stent struts and vessel lumen with high spatial resolution.

The present strategy has several limitations to be clarified. First, this is the first case presentation of the preshaped Y-stent strategy for a true bifurcation lesion in a real-life patient; however, it is still a proof-ofconcept case demonstration. Not until further studies confirm the efficacy and safety of this technique to treat complex bifurcation lesions will the technique be widely accepted as a useful strategy for bifurcation. Second, the safety of ex vivo stent preparation is not known. Adverse effects on the drug and polymer mounted on the stent alloy may be caused by dilating a proximal part of the stent with the remaining distal portion prevented from dilation by being covered with the plastic tube, passing a stylet and the other stent via a stent strut and manually remounting the dilated portion of the stent onto the stent balloon. The presumed disruption of stent components might hamper the expected anti-restenotic effect, eventually raising the possibility of adverse clinical outcomes. We need further investigation by angiographic and clinical follow-up after the proposed Y-stent technique. Finally, in the present case, we selected the LCX as an MV and the LAD as a SB according to the vessel sizes, with the former having a larger diameter of $4.0 \mathrm{~mm}$ compared with the latter diameter of $3.5 \mathrm{~mm}$. We made the decision on the presumption that the strategy might reduce the number of unopposed struts within the proximal MV by dilating the first deployed $3.5 \mathrm{~mm}$ stent with a consequently placed $4.0 \mathrm{~mm}$ stent. We usually, however, select the LAD as an MV and the LCX as a SB in almost all cases in daily clinical practice. Therefore, we need to confirm the feasibility of this technique for 
bifurcation lesions where we regard the LAD as an MV and the LCX as a SB. With all the abovementioned limitations of the present case study, we believe that the preshaped Y-stent strategy lessens the complexity of the conventional Y-stent technique, raising the success rate of the complex two-stent technique and finally contributing to favorable clinical outcomes.

\section{Conclusion}

We demonstrated the first case of a preshaped Y-stent technique for the left main distal bifurcation lesion. This strategy decreases the complexity of the conventional Y-stent technique. The present case is still a proof-of-concept demonstration that needs further investigation.

\section{Acknowledgment}

We would like to express our gratitude to the staff members of our department and to the clinical medical engineering staff and our radiology technicians and nurses, all of whom support our daily clinical practice.

\section{Funding}

None.

Conflict of Interest The authors declare that they have no conflicts of interest.

\section{Author Contribution}

TO and TT: performed the procedure. TO and TT: equally contributed to the paper.

\section{References}

1. Lassen JF, Burzotta F, Banning AP, Lefevre T, Darremont O, Hildick-Smith D, Chieffo A, Pan M, Holm NR, Louvard Y and others. Percutaneous coronary intervention for the left main stem and other bifurcation lesions: 12th consensus document from the European Bifurcation Club. EuroIntervention 2018;13:1540-1553.

2. Brar SS, Gray WA, Dangas G, Leon MB, Aharonian VJ, Brar SK, Moses JW. Bifurcation stenting with drug-eluting stents: a systematic review and meta-analysis of randomised trials. EuroIntervention $2009 ; 5: 475-84$.

3. Hildick-Smith D, Behan MW, Lassen JF, Chieffo A, Lefevre T, Stankovic G, Burzotta F, Pan M, Ferenc M, Bennett L and others. The EBC TWO Study (European Bifurcation Coronary TWO): A Randomized Comparison of Provisional T-Stenting Versus a Systematic 2 Stent Culotte Strategy in Large Caliber True Bifurcations. Circ Cardiovasc Interv 2016;9.

4. Ford TJ, McCartney P, Corcoran D, Collison D, Hennigan B, McEntegart M, Hildick-Smith D, Oldroyd KG, Berry C. Single- Versus 2-Stent Strategies for Coronary Bifurcation Lesions: A Systematic Review and Meta-Analysis of Randomized Trials With Long-Term Follow-up. J Am Heart Assoc 2018;7.

Figure legends

Figure 1. Ex vivo preparation of the preshaped Y-stent system

Figure 2. The preshaped Y-stent system delivered to the left main distal bifurcation

Figure 3. LAD stenting

Figure 4. LCX stenting

Figure 5. Proximal optimization therapy

Figure 6. Kissing balloon technique

Figure 7. Final angiogram

Video legends 
Video 1. Ex vivo preparation of the preshaped Y-stent system

We pulled a plastic tube cover a distance of $5 \mathrm{~mm}$ over the stent for the LAD, which we estimated was equal to the length of the left main part of the Y-stent. Next, we gradually inflated the stent system with the plastic tube firmly held by hand to prevent movement of the tube on the stent. Six-atmosphere inflation was enough to dilate the uncovered stent struts outside the plastic tube.

Video 2. Ex vivo preparation of the preshaped Y-stent system

After passing a stylet from the most distal strut of the dilated portion of the LAD stent through the proximal stent ostium, the stent for the LCX was delivered on the stylet from the ostium of the dilated LAD stent through the strut at the stylet exit. Finally, we gently remounted the dilated portion of the LAD stent on the stent balloon.

Video 3. Delivery of the preshaped Y-stent system to the distal left main bifurcation lesion

Video 4. Deployment of the preshaped Y-stent system

We placed the LAD stent and then deployed the LCX stent, smoothly completing the formation of the Y-like configuration.

\section{Supplementary Figure legends}

Supplementary Figure 1. Pulling a plastic tube cover $5 \mathrm{~mm}$ over the LAD stent yielded a distance equal to the length of the left main part of the Y-stent.

Supplementary Figure 2. Gradual inflation of the stent system with the plastic tube firmly held by hand to prevent its movement on the stent.

Supplementary Figure 3. Dilation of the uncovered stent struts outside the plastic tube. We passed a stylet from the most distal stent strut (arrow) through the proximal stent ostium (arrowhead).

Supplementary Figure 4. The LCX stent was delivered on the stylet from the ostium of the dilated LAD stent.

Supplementary Figure 5. The LCX stent was delivered from the ostium of the dilated LAD stent (arrowhead) through the strut at the stylet exit (arrow).

Supplementary Figure 6. Gentle remounting of the dilated portion of the LAD stent on the stent balloon.

Supplementary Figure 7. Final IVUS at the LAD ostium.

Supplementary Figure 8. Final IVUS at the LCX ostium.

Supplementary Figure 9. Final IVUS at the left main trunk. 



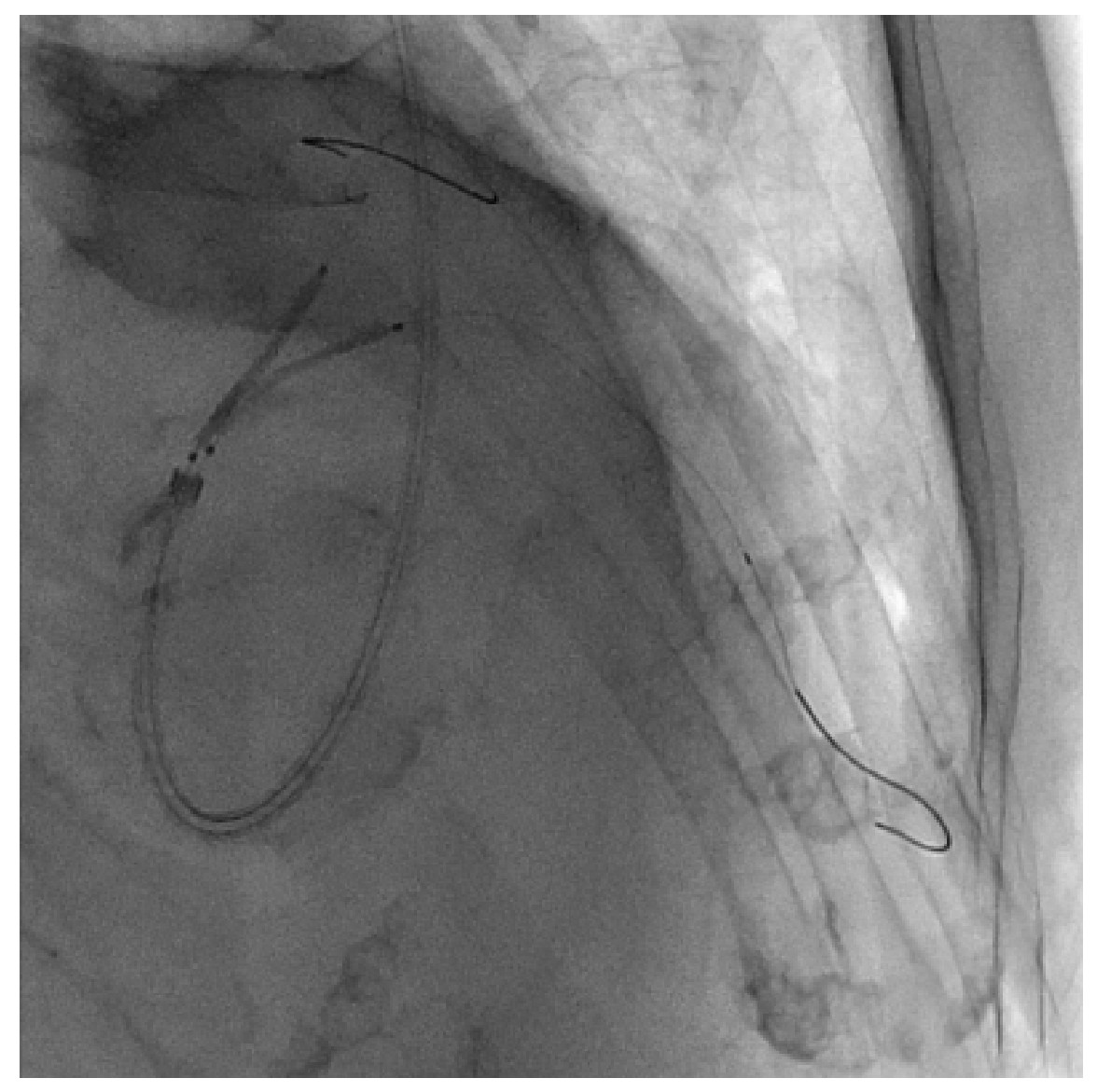




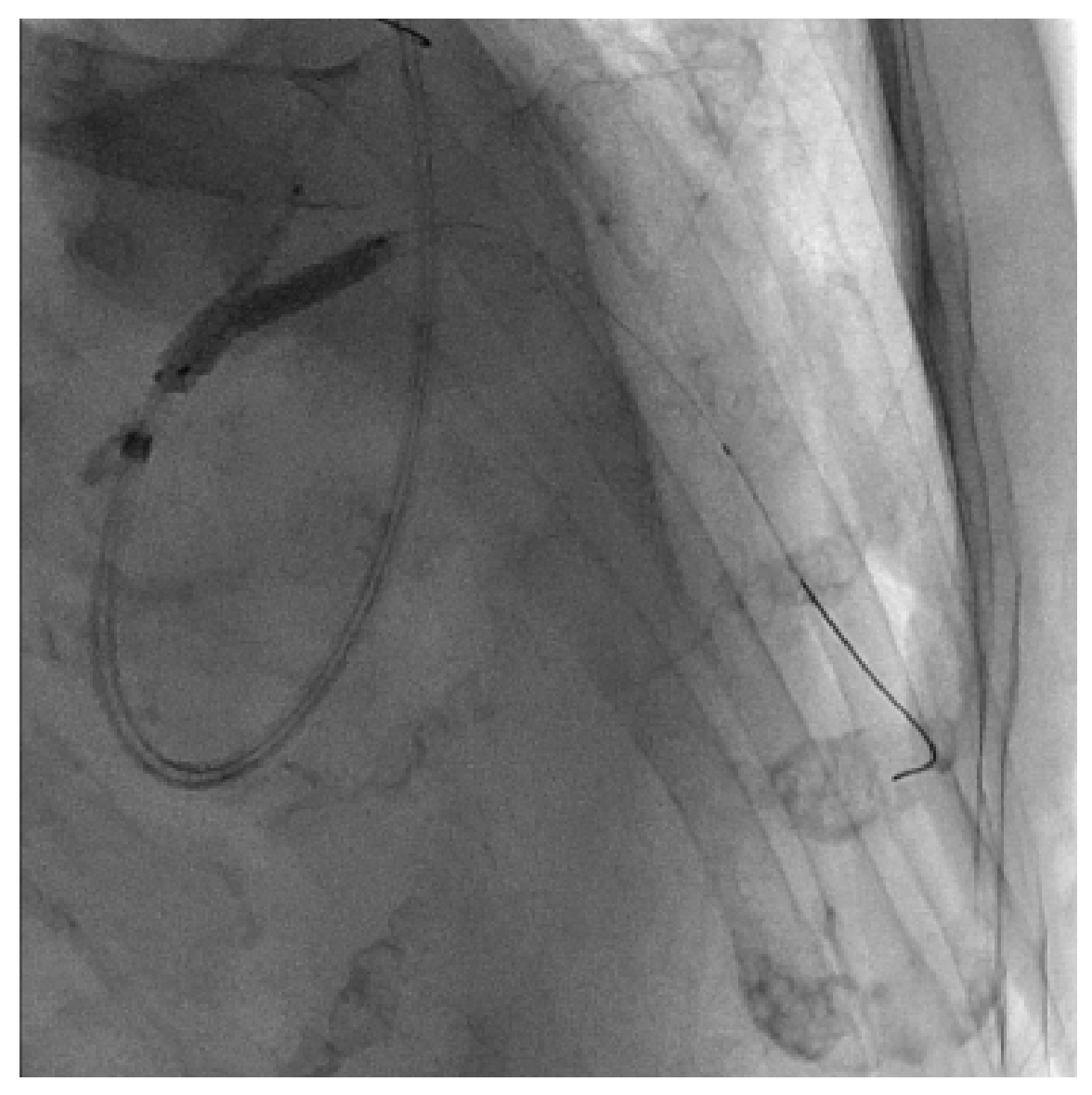




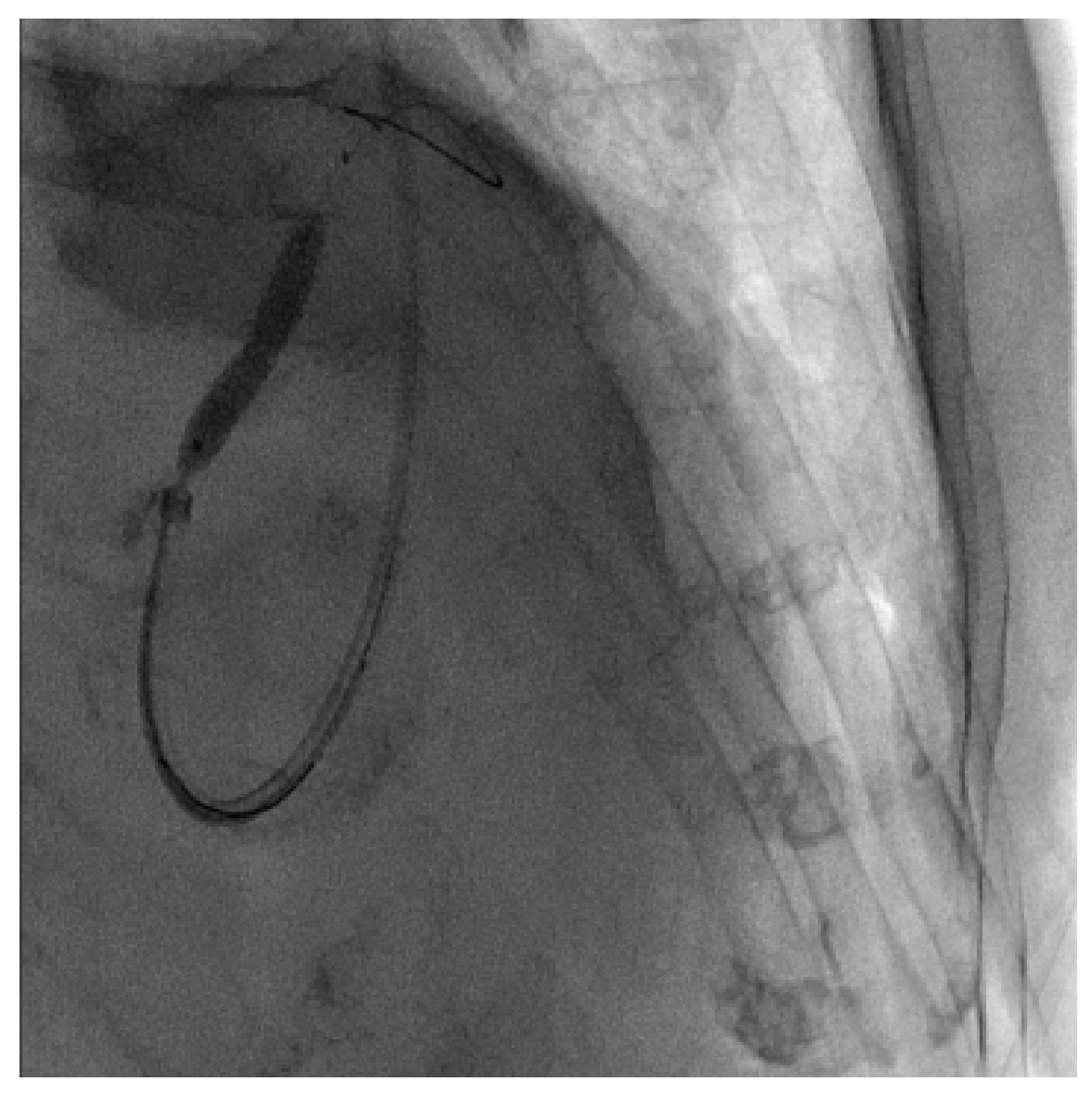




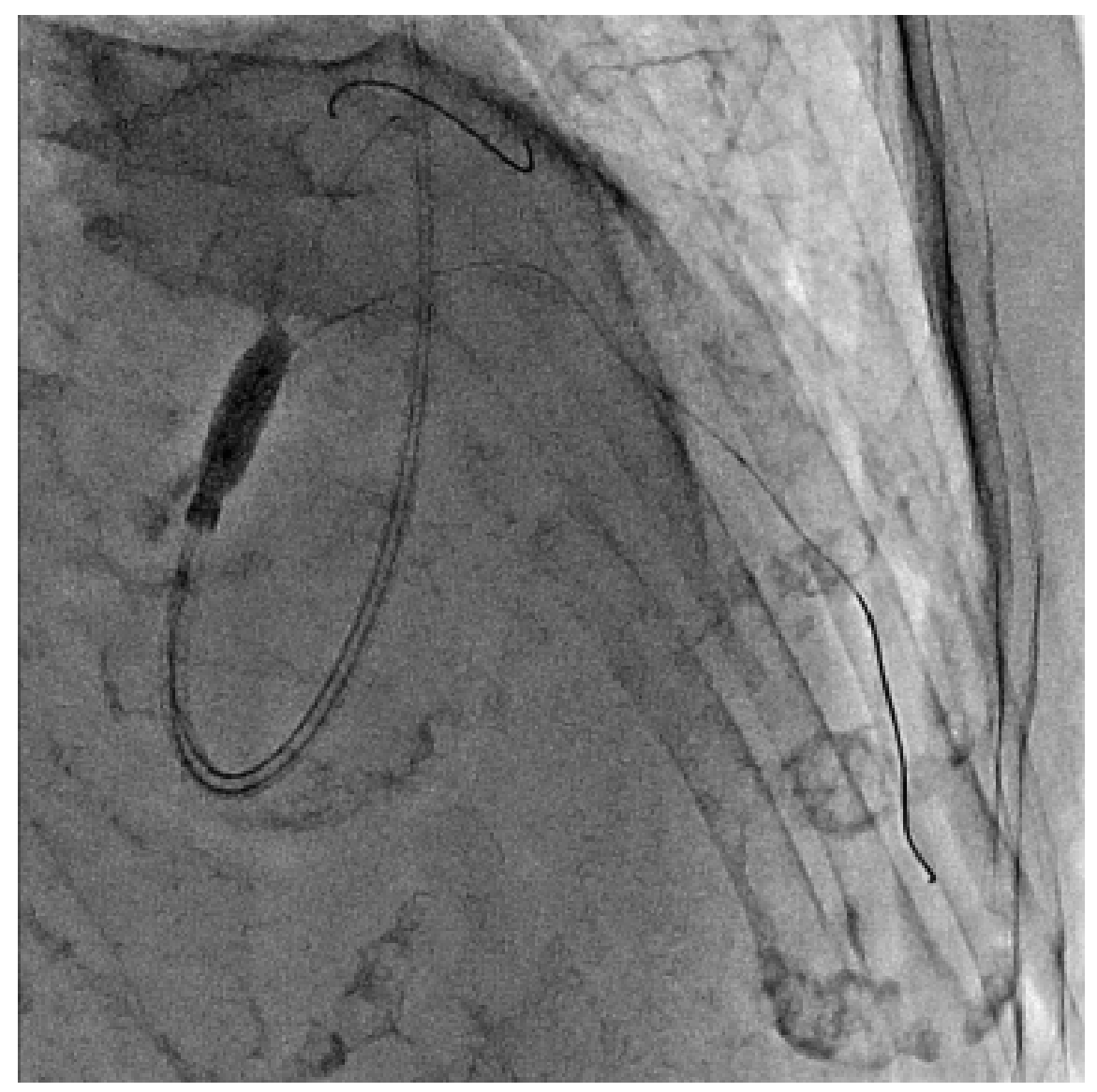




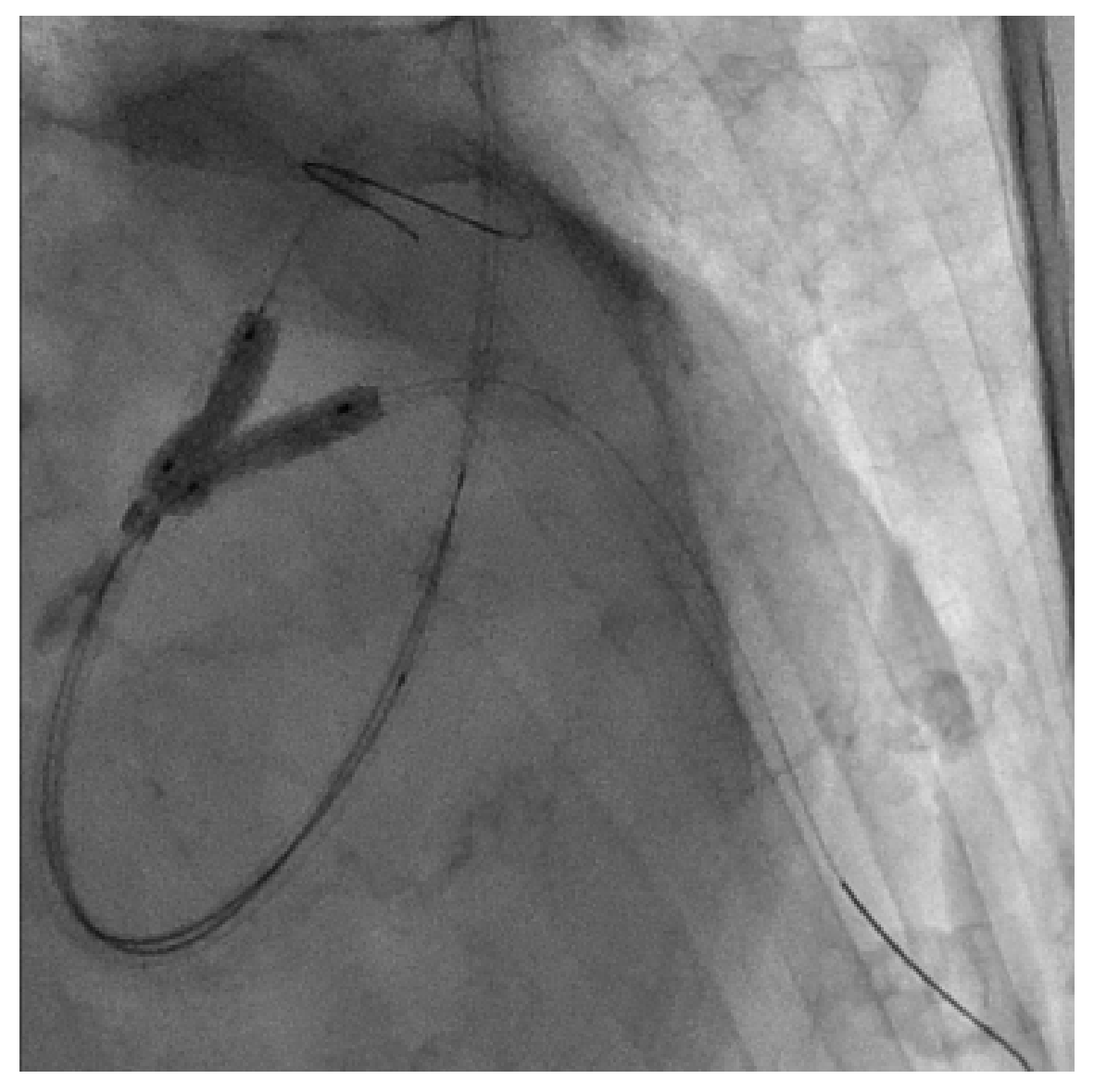




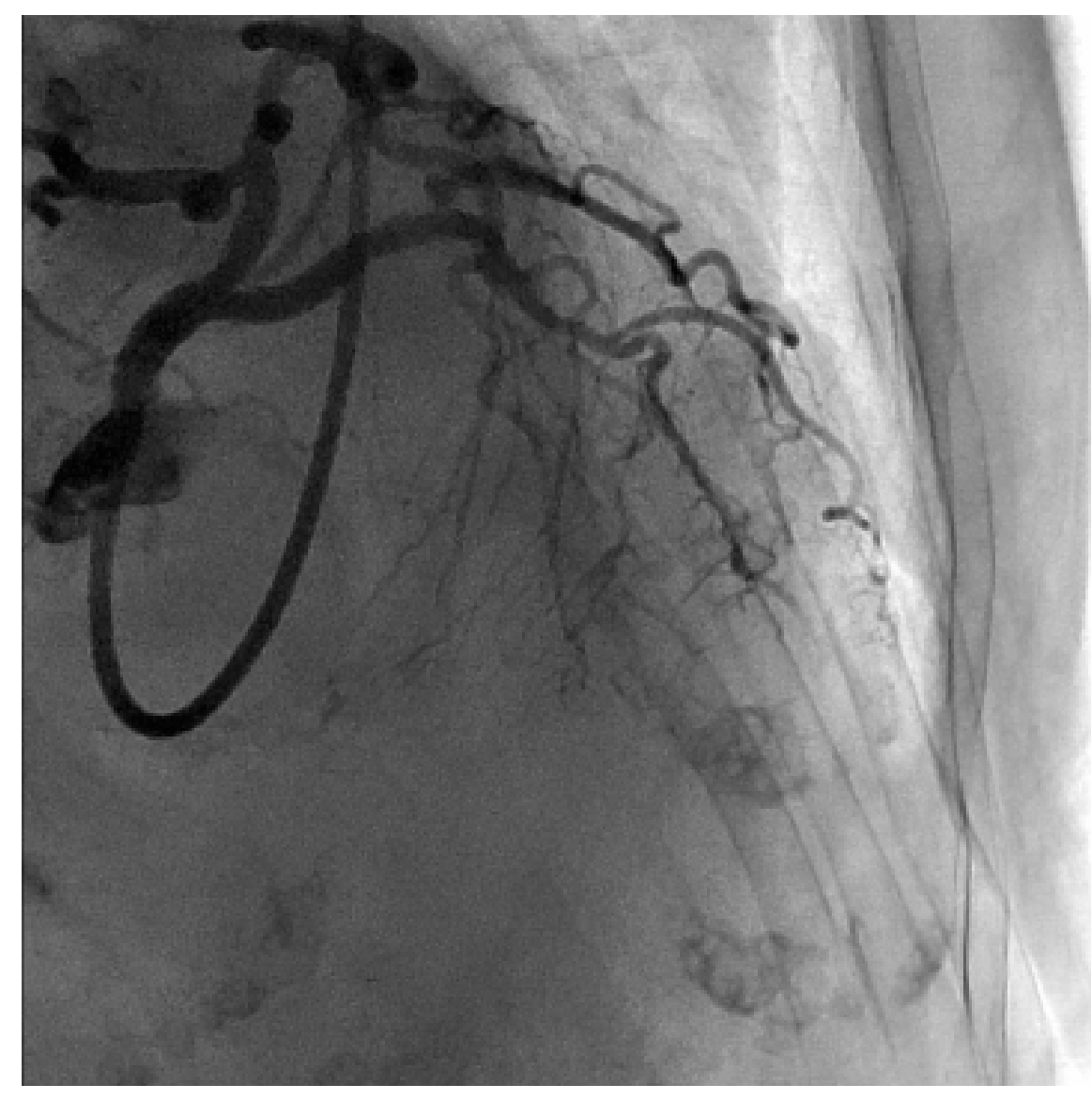

